\title{
Analysis of Liver Fibrosis Degree with APRI Score and FIB-4 Index on Patients with Non-Alcoholic Fatty Liver Disease
}

\author{
Gillian Elvira Seipalla ${ }^{1}$, Nurahmi ${ }^{2}$, Ibrahim Abd Samad ${ }^{3}$ \\ ${ }^{1}$ Medical Doctor Specialist, Study Program of Clinical Pathology, Faculty of Medicine, Hasanuddin University/Dr. Wahidin Sudirohusodo \\ Hospital, Makassar, Indonesia. E-mail:anggavril01@gmail.com \\ ${ }^{2}$ Department of Clinical Pathology, Faculty of Medicine, Hasanuddin University/Daya Hospital, Makassar, Indonesia \\ ${ }^{3}$ Department of Clinical Pathology, Faculty of Medicine, Hasanuddin University/Ibnu Sina Hospital, Makassar, Indonesia
}

\section{ABSTRACT}

Liver fibrosis is the accumulation of Extracellular Matrix Protein (EMP) scar tissue after acute or chronic liver injury. Liver biopsy is the gold standard test for evaluating liver fibrosis in patients with Non-Alcoholic Fatty Liver Disease (NAFLD). This diagnostic method is invasive, painful, and complicated in rare cases; thus the noninvasive methods such as laboratory tests and radiology had been proposed to assess liver fibrosis in NAFLD and are expected to overcome the limitations of liver biopsy. This method consists of aspartate Aminotransferase to the Platelet Ratio Index (APRI) and fibrosis-4 (FIB-4).This research was retrospective cross-sectional performed at Dr. Wahidin Sudirohusodo Hospital Makassar involving 63 subjects from January to June 2018. The correlated variables were presented in categorical and numerical data and the Pearson correlation test was used to determine the compatibility of APRI score and FIB-4 index with Fibroscan result in patients with NAFLD. A highly significant positive correlation $(p<0.001)$ was found between APRI score and Fibroscan (correlation value $=45.8 \%$ ), APRI score and FIB-4 index (correlation value $=91.8 \%$ ), FIB-4 index and Fibroscan (correlation value $=47.6 \%$ ). The score of Aminotransferase to platelet ratio index score and FIB-4 index could be an alternative method instead of a liver biopsy to predict the degree of fibrosis in patients with NAFLD.

Keywords: Liver fibrosis, APRI score, FIB-4 index

\section{INTRODUCTION}

Non-alcoholic fatty liver is defined as an accumulation of fat in the liver, characterized by more than $5 \%$ hepatocytes containing intracellular triglycerides or minimum $5 \%$ fat in volume or liver weight in patients consuming less than $30 \mathrm{~g}$ ( 3 units) of alcohol a day for male and less than $20 \mathrm{~g}$ (2 units) of alcohol a day for female. One unit of alcohol $(10 \mathrm{~g})$ is equal to one glass of beer $(250 \mathrm{~mL})$, one glass of wine $(200 \mathrm{~mL})$ or one whiskey glass $(30 \mathrm{~mL})$. Globally, non-alcoholic fatty liver disease is the most common type of chronic liver disease among adults. It occurs $14 \%-30 \%$ in common population. ${ }^{1,2}$ The prevalence of non-alcoholic fatty liver disease ranges from $15-20 \%$ in the adult population in the United States, Japan, and Italy. It is estimated that $20-30 \%$ are in a more severe phase (non-alcoholic steatohepatitis). ${ }^{3}$

A study of obese populations in developed countries showed $60 \%$ simple fatty liver, $20-25 \%$ Non-Alcoholic Steatohepatitis(NASH) and 2-3\% cirrhosis. The prevalence of Non-Alcoholic Fatty Liver Disease (NAFLD) in an Indonesian urban population was estimated in one study approximately $30 \% .{ }^{4}$
In patients, morbidity and mortality with NAFLD are related to the process of NASH development into fibrosis lesions and cirrhosis. Liver fibrosis is an accumulation of Extracellular Matrix Protein (EMP) scar tissue after acute or chronic liver injury. Histopathology of liver tissue is a gold standard for evaluating liver fibrosis in non-alcoholic fatty liver patients; however, this diagnostic method is invasive, painful, and causes severe complications in some rare cases. The preferred methods such as non-invasive laboratory and radiological examinations to assess liver fibrosis in PPHNA has been developed over the past decade and are expected to overcome the limitations of liver biopsy. This method consists of Aspartate Aminotransferase (AST)/Alanine Aminotransferase (ALT) Ratio, AST to Platelet Ratio Index (APRI) and fibrosis-4 (FIB-4).

Risk factors for non-alcoholic fatty liver disease include age, male sex (in Asians), obesity, and insulin resistance. In most studies in western countries, the average age of patients with non-alcoholic fatty liver disease was 50-55 years, higher than in Asian countries with an average age of 45 years. In females, in contrast with Asian countries. ${ }^{5,6}$ 
FIB-4 index was firstly introduced by Sterling et al. in 2006. It is a non-invasive method with $90 \%$ sensitivity, 64\% specificity and 98\% Negative Predictive Value (NPV). Its sensitivity and NPV is higher than other noninvasive methods. ${ }^{4,5}$

$$
\text { FIB-4 Index }=\text { Age }(y r) \times \frac{A S T \text { (IU/L) }}{\text { Platelet Count }\left(10^{9} / \mathrm{L}\right)} \times \sqrt{A L T(I U / L)}
$$

Aspartate aminotransferase-plateletratio score is another method to detect non-invasive liver fibrosis. It was firstly introduced in 2003 by Wai et al. with 30\% sensitivity and $93 \%$ specificity.

Aspartate aminotransferase and platelet count are indirect indicators because they are not directly associated with liver fibrosis, but they are associated with the occurrence of liver dysfunction.

$$
\text { APRI score }=\frac{A S T(I U / L)}{\text { Platelet Count }\left(10^{9} / L\right)} \times 100
$$

This study was aimed to analyze the compatibility of FIB-4 Index, APRI score and Fibroscan to determine the degree of liver fibrosis in patients with NAFLD in Indonesia, especially in Makassar.

\section{METHODS}

This research was a retrospective study with a cross-sectional method by taking secondary data of non-alcoholic fatty liver patients on the Medical Record Installation of Dr. Wahidin Sudirohusodo Hospital Makassar from January 2018 to June 2018.

The population was medical record data of non-alcoholic fatty liver patients at Dr. Wahidin Sudirohusodo Hospital Makassar. Samples were patients among the population who were examined the AST and ALT levels. Platelet count was measured in the Laboratory Installation and Fibroscan examination was performed in the Gastroenterohepatology Sub division of Dr. Wahidin Sudirohusodo Hospital. The inclusion criteria were patients diagnosed with non-alcoholic fatty liver by internist based on history, physical and additional examinations, while patients with the incomplete medical record were excluded. Data of non-alcoholic fatty liver was classified based on Fibroscan results in some fibrosis degree, such as normal (F0), mild (F1), moderate (F2), severe (F3), and cirrhosis (F4).

FIB-4 index was calculated based on Sterling's formulation, while the APRI score was calculated based on Wais formulation. Data were statistically analyzed using SPSS version 22 with the Pearson correlation test to determine the correlation between non-invasive markers and Fibroscan. Ethical approval of this study was obtained from the Health Research Ethics Committee of the Faculty of Medicine, Hasanuddin University/Central General Hospital of Dr. Wahidin Sudirohusodo with number 571/H4.8.8.5.31/PP36-KOMETIK/2018.

\section{RESULTS AND DISCUSSION}

Based on the data collection during the period of January 2018 - June 2018, 63 patients diagnosed with non-alcoholic fatty liver were involved, with characteristics as shown in Table 1. It shows that subjects consisted of 37 female (58.7\%) and 26 males (41.3\%). In the age group of 40-49 years, 26 people were diagnosed with non-alcoholic liver disease (41.3\%).

Table 1. Distribution of subjects characteristics $(n=63)$

\begin{tabular}{llll}
\hline Variable & & $\mathbf{n}$ & $\%$ \\
\hline Gender & Males & 26 & 41.3 \\
& Females & 37 & 58.7 \\
Ages & $<40$ years & 18 & 28.6 \\
& 40-49 years & 26 & 41.3 \\
& $>=50$ years & 19 & 30.2 \\
\hline
\end{tabular}

Source: Secondary data

Table 2. Descriptive statistic of laboratory result $(n=63)$

\begin{tabular}{lccc}
\hline Variable & Ranges & Mean & SD \\
\hline APRI score & $0.09-1.85$ & 0.36 & 0.33 \\
Fibroscan & $4.20-9.70$ & 6.31 & 1.41 \\
FIB-4 & $0.24-4.72$ & 1.07 & 0.80 \\
\hline
\end{tabular}

Table 3. Correlation between APRI score, Fibroscan, and FIB-4

\begin{tabular}{llrr}
\hline Variable & Statistics & Fibroscan & \multicolumn{1}{c}{ FIB-4 } \\
\hline APRI score & Pearson correlation (R) & 0.677 & 0.958 \\
& P & $0.000^{\star}$ & $0.000^{\star}$ \\
& N & 63 & 63 \\
Fibroscan & Pearson correlation (R) & & 0.690 \\
& P & & $0.000^{\star}$ \\
& N & & 63 \\
\hline
\end{tabular}

$\mathrm{P}^{*}$ : Pearson's correlation test 
Table 2 shows the clinical and laboratory examinations such as the APRI score which ranged between $0.09-1.85$ with an average of 0.36 , Fibroscan ranged between 4.20 - 9.70 with a mean of 6.31 , while FIB-4 ranged between 0, 24-4.72 with an average of 1.07 .
To determine the correlation between the three parameters, Pearson's correlation test was used. Significant positive correlation was found between APRI scores and Fibroscan ( $p<0.001$ ) with a magnitude of correlation of $0.6772=0.458$ or $45.8 \%$ (Figure 1).

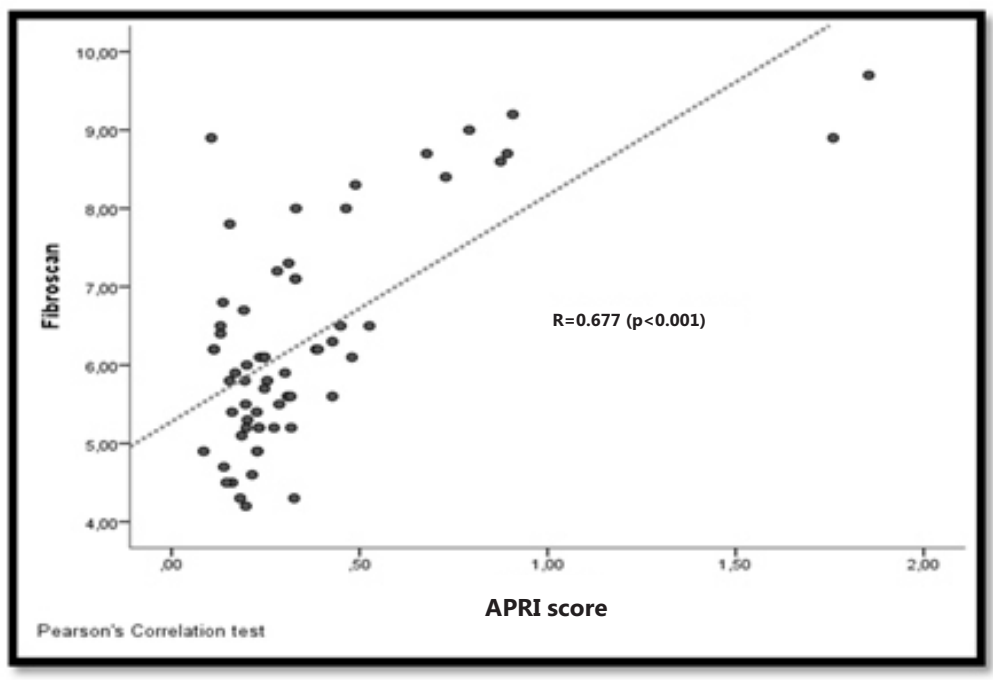

Figure 1. Correlation between APRI score and Fibroscan

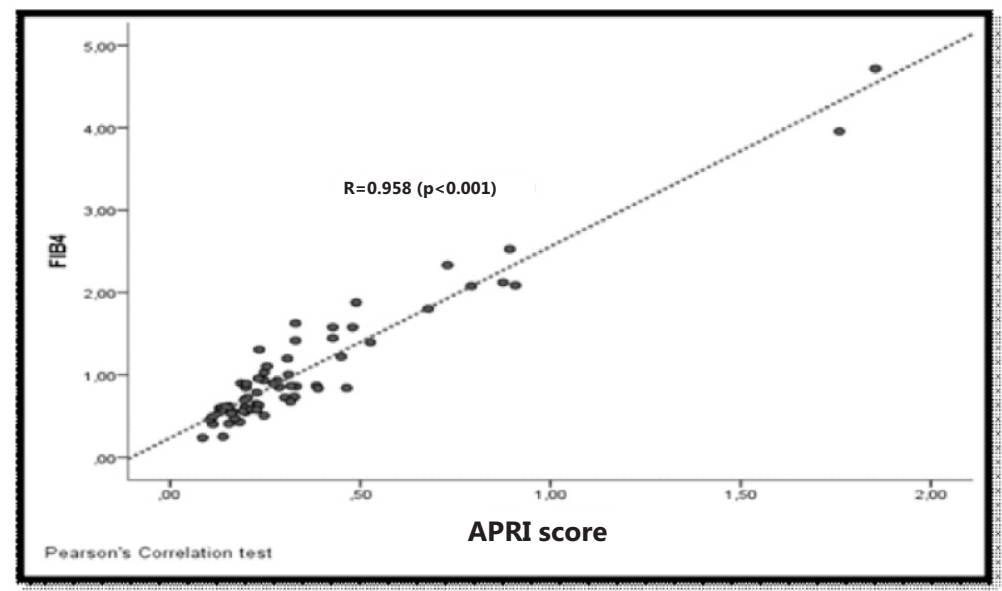

Figure 2. Correlation between APRI score and FIB-4

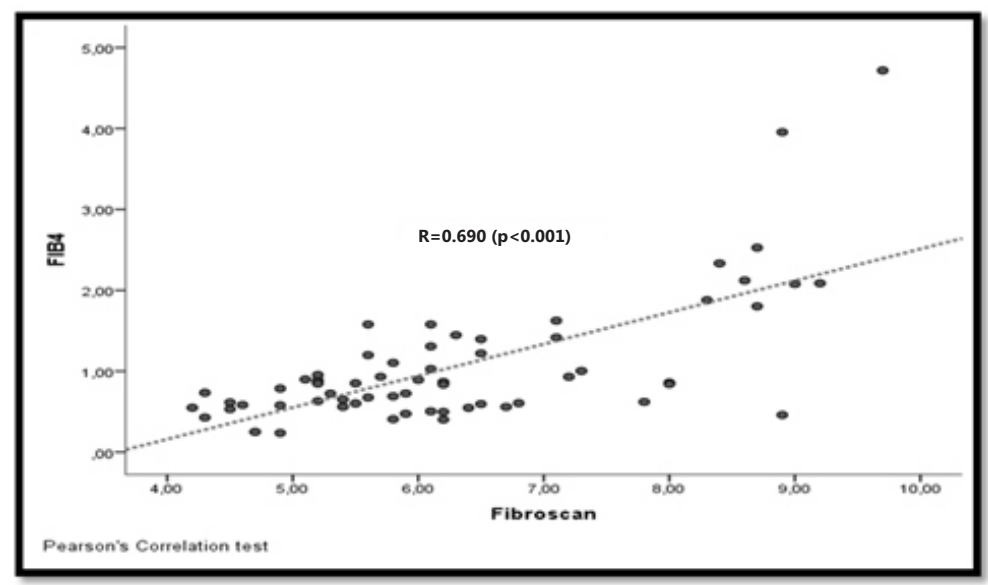

Figure 3. Correlation of Fibroscan and FIB-4 
A significant positive correlation was found between APRI scores and FIB-4 $(p<0.001)$. The magnitude of the correlation between the two parameters was $0.9582=0.918$ or $91.8 \%$ (Figure 2).

Significant positive correlation was found between Fibroscan and FIB-4 ( $p<0.001)$. The magnitude of the correlation between the two parameters was $0.6902=0.476$ or $47.6 \%$ (Figure 3 ).

Based on data collected during the period of January 2018 June 2018, 63 patients diagnosed with non-alcoholic fatty liver were involved as subjectswith characteristics as shown in Table 1. It showed that there were more females 37 people (58.7\%) than males (41.3\%). It was found in the age group of 40-49 years that there were more than 26 people who were diagnosed with non-alcoholic liver disease (41.3\%).

In this study, distribution of gender and age was in line with study by Josida et al. in 2016 suggesting that the incidence of non-alcoholic fatty liver disease was more common in females than males, with the highest average age ranged between 40-49 years which is the productive period frequently exposed to risk factors of non-alcoholic fatty liver such as obesity and diabetes mellitus. Obesity is the most important risk factor for the fatty liver disease. The prevalence of fatty liver disease is 4.6 times greater in the obese population.?

Table 3 shows a significant positive correlation $(p<0.001$ ) between FIB-4 index, APRI score and Fibroscan. It was suggested that the increase in the FIB-4 index and APRI score was directly proportional to Fibroscan; the higher level of each marker led to amore severe degree of liver fibrosis. This was consistent with the previous hypothesis suggesting that the increase in each marker level was proportional to the severity of liver fibrosis degree. Formulation of FIB-4 index and APRI score uses AST and platelet as an indicator; thus the increase of AST levels and the decrease of platelet count are in line with the severity of liver fibrosis. The increase of AST levels is associated with hepatocyte damage which triggers the release of AST from mitochondria and the decrease of AST clearance due to liver fibrosis. The decrease of platelet counts is due to decreased production of thrombopoietin by damaged hepatocytes. $^{8}$

In a state of advanced liver fibrosis to portal hypertension, platelets will undergo sequestration and destruction in the lymph. In addition to AST and platelet, ALT levels are added to the formulation of the FIB-4 index. ALT is an intracellular enzyme located in the cytoplasm of hepatocytes which will be released when mild hepatocytes damage occurs, in contrast to AST enzyme concentrated in hepatocytes mitochondria; thus the release of AST generally occurs in advanced hepatocytes damage. . $^{80}$

\section{CONCLUSION AND SUGGESTIONS}

As result of this study, it was concluded that the APRI score and FIB-4 index can be taken into consideration to predict the degree of fibrosis in patients with non-alcoholic fatty liver disease. Therefore, it was expected to be a reference in determining diagnosis and follow-up therapy, especially in areas with no Fibroscan facilities. It was recommended to perform further research based on the degree of fibrosis.

\section{REFERENCES}

1. Aslan A, Turrel D,Ashlan M, Tuney D. Effect of non-alcoholic fatty liver disease on the hepatic vein and artery. Research Article, J Med Diagn Meth, 2014; 3(1): 1-4.

2. Hassan K, Bhalla $V$, El Regal $M E, A-K a d e r ~ H H$. Non-alcoholic fatty liver disease: A comprehensive review of a growing epidemic. World Journal of Gastroenterology, 2014; 12082-101.

3. Buzzeti E, Rosa L,De Luca L, Lombardi L. Non-invasive assessment of fibrosis in patients with non-alcoholic fatty liver disease. International Journal of Endocrinology, 2015; XII: 1-4.

4. Ferrel L. Update on staging of fibrosis and cirrhosis. University of California, American Journal of Pathology, 2013; 137(1): 1-22.

5. Fallatah $\mathrm{H}$. Non-invasive biomarkers of liver fibrosis. Advance in Hepatology Journal, 2014; 1: 1-15.

6. Wilkins $T$, Altaf $T$, Iryna $H$, Robert RS. Non-alcoholic fatty liver disease: Diagnosis and management. American Family Physician, 2013; 88(1): 35-42.

7. Surastik A, Ajay K, Duseja. Non-alcoholic fatty liver disease: East versus West. Journal of Clinical and Experimental Hepatology, 2012; 2(2): 122-134.

8. Mustakin. Analisis derajat fibrosis hati dengan Fibroscan, FIB-4 indeks, King score, dan APRI score pada pasien penyakit hepatitis kronis. Departemen Ilmu Patologi Klinik. Makassar, Universitas Hassanudin. 2015; 1-10.

9. Nakajima A, Yoshio S,Yoshio S, Hyogo H, Tanaka S, Ono $\mathrm{M}$, et al. Non-invasive scoring systems for predicting NASH in Japan: Evidence from Japan Study Group of NAFLD. Integrative Molecular Medicine, Integrative Molecular Medicine Journal, 2015; 2(2): 145-149.

10. Becker L. Evaluation and staging of liver fibrosis. Journal of Hepatology, 2015; 2(2): 1-16. 